\title{
Frameless
}

Volume 1 | Issue 1

Article 20

November 2019

\section{Experimental Analysis of Spatial Sound for Storytelling in Virtual Reality}

Saylee Bhide

Rochester Institute of Technology, smb6390@rit.edu

Elizabeth Goins

Rochester Institute of Technology, esggsh@rit.edu

Joe Geigel

Rochester Institute of Technology, jmg1590@rit.edu

Follow this and additional works at: https://scholarworks.rit.edu/frameless

\section{Recommended Citation}

Bhide, Saylee; Goins, Elizabeth; and Geigel, Joe (2019) "Experimental Analysis of Spatial Sound for Storytelling in Virtual Reality," Frameless: Vol. 1: Iss. 1, Article 20.

DOI: 10.14448/Frameless.01.007

Available at: https://scholarworks.rit.edu/frameless/vol1/iss1/20

This Article is brought to you for free and open access by RIT Scholar Works. It has been accepted for inclusion in Frameless by an authorized editor of RIT Scholar Works. For more information, please contact ritscholarworks@rit.edu. 


\title{
FRAMEIESS
}

\section{Experimental Analysis of Spatial Sound for Storytelling in Virtual Reality}

\author{
Saylee Bhide \\ Department of Computer Science \\ Rochester Institute of Technology
}

\author{
Elizabeth Goins \\ Department of Performing Arts and Visual Culture \\ Rochester Institute of Technology
}

\author{
Joe Geigel \\ Department of Computer Science \\ Rochester Institute of Technology
}

\begin{abstract}
Virtual Reality leverages our cognitive and perceptual abilities to provide immersive experiences that recreate both the visual and aural elements of real spaces with a high degree of realism making it a suitable delivery platform for conveying narratives through games and films. Spatial sound is useful in enhancing immersion and presence of the user in a virtual world. This audio design allows the game designer to place audio cues that appropriately match with the visual cues in a virtual game environment. These localized audio cues placed in a story based game environment also help to evoke an emotional response from the user and construct the narrative of the game by capturing the user's attention towards the guiding action events in the game. Our work currently involves a thorough literature study on the significance of debating the usefulness of spatial sound. Our future work involves conducting a user study for analyzing the same i.e., understanding how spatial sound improves user performance and user experience in a virtual game environment. Furthermore, with the help of the relevant subjective and objective inferences that will be collected from the user study conducted on four different evaluation models, our work will also analyze and establish the potential of spatial sound as a powerful storytelling tool in a virtual game environment.
\end{abstract}


Vol. 1, No. 1 - 2019

Frameless

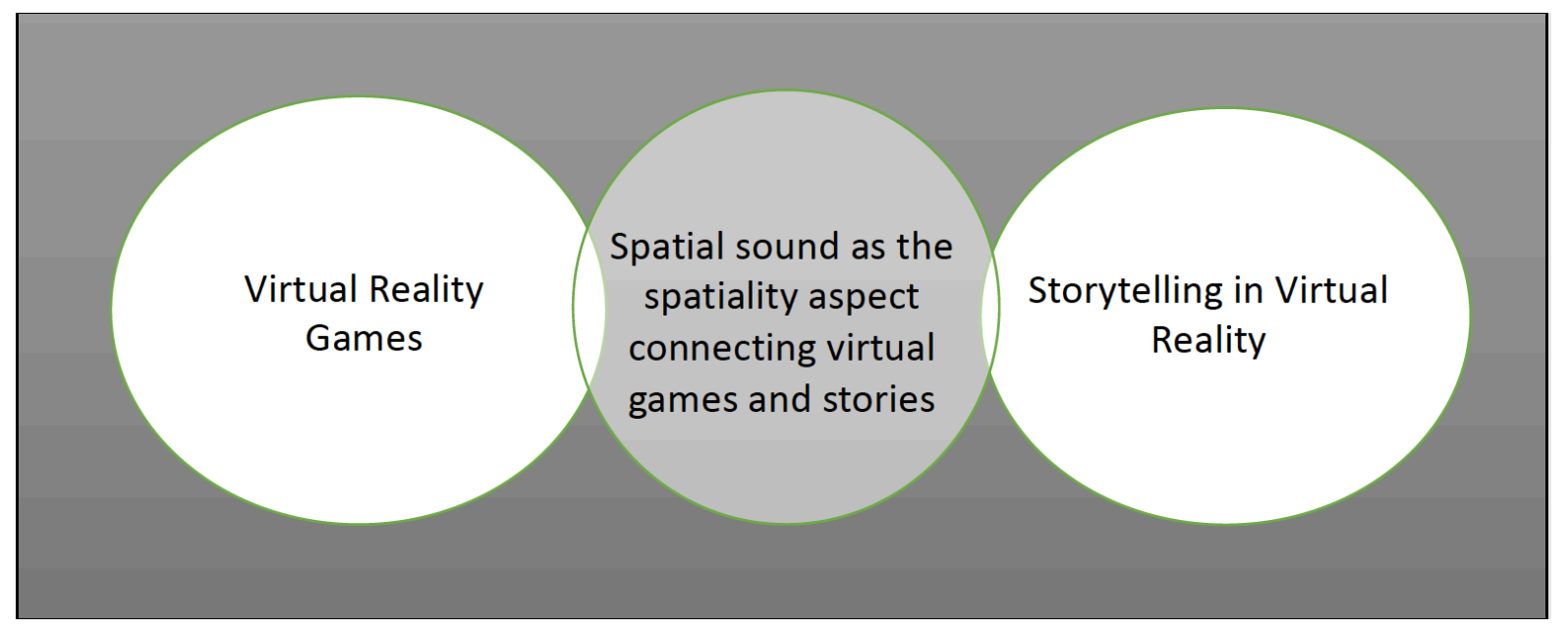

Fig 1: Depiction of the approach for exploring the problem space.

Fall 2018. Photo Credit: Saylee Bhide

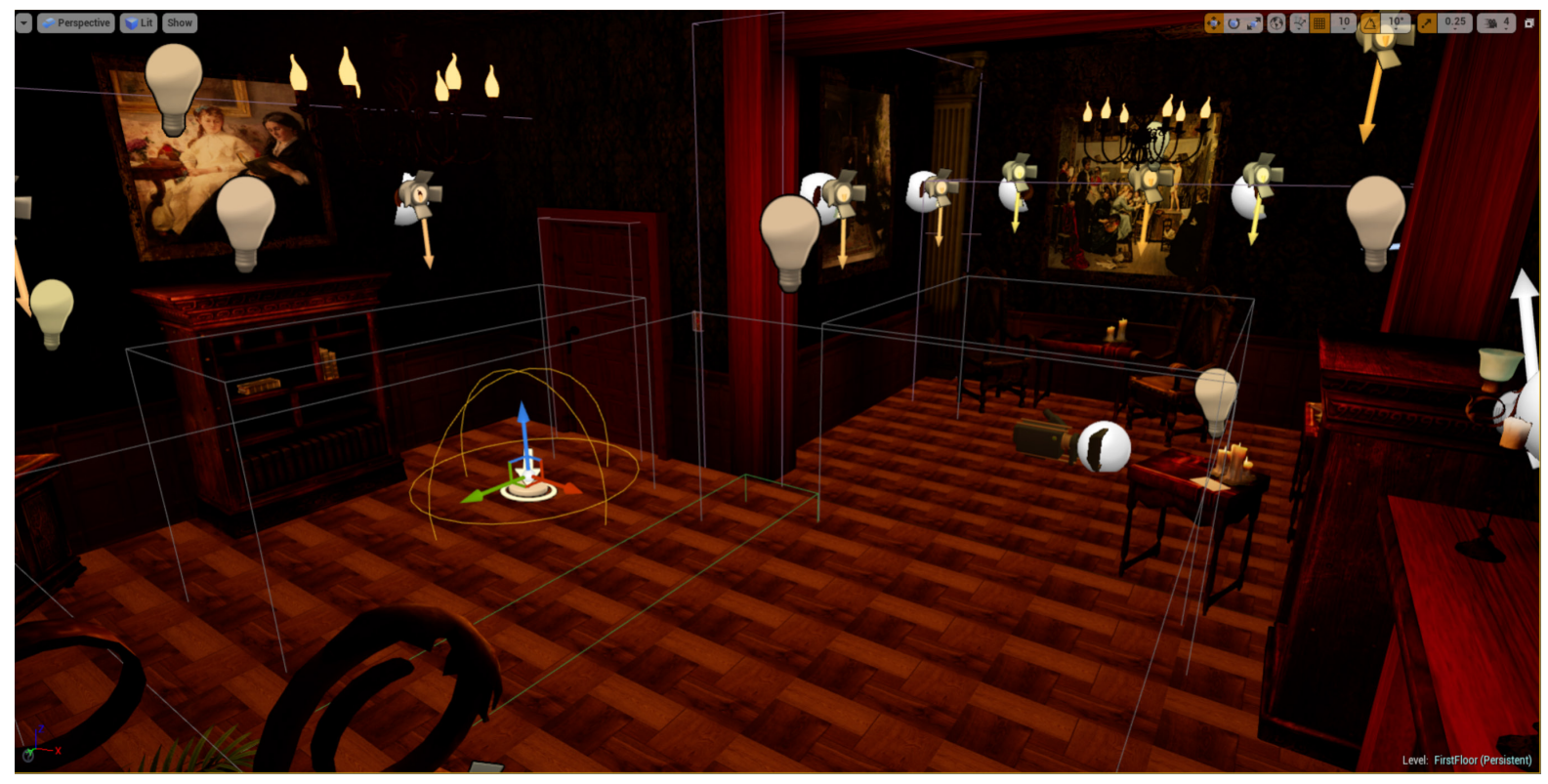

Fig 2: Screenshot of the introductory room in the puzzle, Charlotte.

Spring 2019. Photo Credit: Saylee Bhide 


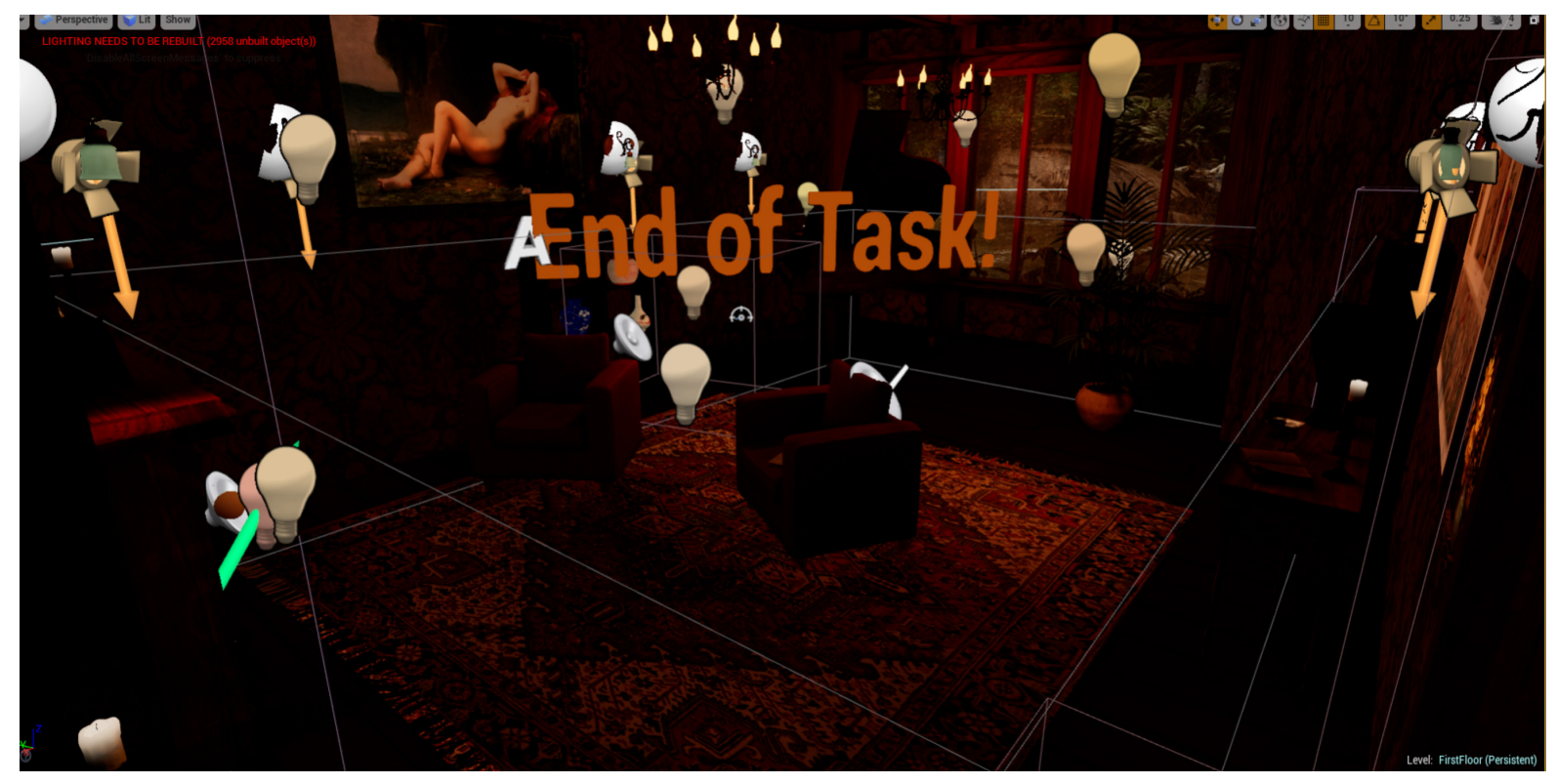

Fig 3: Screenshot of the final room in the puzzle, Charlotte. Spring 2019. Photo Credit: Saylee Bhide

Following is the Google Drive link to download the video of the puzzle, Charlotte, completed in Spring 2019.

https://drive.google.com/file/d/1TIoFLxcKE5qoG01h7vBti6PxTsEwz2jG/ view? usp $=$ sharing 